\title{
The interpretation of the degenerative changes in the intralaminar nuclei of the thalamus
}

\author{
T. P. S. POWELL AND W. M. COWAN \\ From the University of Oxford, Department of Human Anatomy, \\ South Parks Road, Oxford
}

Although the intralaminar nuclei of the thalamus are now known to have an organized, extrathalamic projection through the internal capsule (Droogleever Fortuyn, and Stefens, 1951; Nashold, Hanbery, and Olszewski, 1955; Powell and Cowan, 1956), the precise mode of termination of the efferents from these nuclei remains problematical. The experimental evidence bearing upon the projection of these nuclei which is available at present permits of two possible interpretations. Because the cells of these nuclei undergo a certain degree of cellular degeneration as the result of large lesions of the cerebral cortex it has been suggested that the intralaminar nuclei send their axons, or axon collaterals, directly to the cortex. On the other hand, because these nuclei show much more severe degeneration after lesions involving the internal capsule and striatum it seems reasonable to conclude that the striatum is in some way implicated in their projection.

As it has become apparent that the experimental evidence upon which both of these views are based is inconclusive, we have carried out a number of different types of experiment in an attempt to distinguish between them. In view of certain technical difficulties, however, and because of problems associated with the interpretation of cellular degeneration in these nuclei, the present experiments, by themselves, have also failed to give an unequivocal answer to this question. However, it is considered worthwhile to place the observations on record as they suggest that the relationship of the intralaminar nuclei to both the cortex and the striatum may be different from that which has previously been considered.

\section{MATERLAL AND METHODS}

The brains of 39 monkeys (Macaca mulatta) were available for this study. In 26 of these brains lesions had been placed in either the caudate nucleus or in the putamen. Most of these lesions were placed stereotactically, but in a few the lesions in the caudate nucleus were made under direct vision through an incision in the rostral part of the corpus callosum. In three brains stereotactic lesions had been placed in the thalamus for another investigation, and the remaining brains had lesions in various parts of the cerebral cortex. All the animals with striatal lesions and four with cortical ablations were allowed to survive for periods ranging between four and 18 weeks. These brains were fixed either in $70 \%$ alcohol and $2 \%$ acetic acid or in $10 \%$ formolsaline, and a regular one in five or one in 10 series of sections throughout most of thr: cerebral hemisphere was mounted and stained with thionine. The remaining animals with cortical lesions survived for between one and two weeks, were perfused with $10 \%$ formol-saline and after a further period of fixation were sectioned on a freezing microtome and stained according to the method of Nauta (1957).

\section{RESULTS}

The first group of experiments to be described was undertaken in order to determine the severity of the cellular changes in the intralaminar nuclei after large cortical lesions. As we are not so much interested in the distribution of the degeneration in the intralaminar nuclei as in the qualitative features of the degeneration, only three experiments will be described. In experiment OM40 almost all of the cortex rostral to the central sulcus has been removed. The only parts of the frontal lobe which have not been directly damaged are a small strip of area 4 along the anterior bank of the central sulcus and the most caudal part of the orbital surface (parts of areas 13 and 14) (Fig. 1). Although in places the lesion extends deeply into the subcortical white matter surrounding the striatum, at no point has it encroached upon the caudate nucleus, putamen, or internal capsule. Following this extensive lesion there are marked changes in the thalamus involving the anterior, medial, and ventral groups as well as the intralaminar nuclei. The severity of the cellular changes in the intralaminar nuclei is quite different from those in the principal nuclei and, consequently, they stand out clearly. In the affected parts of the 


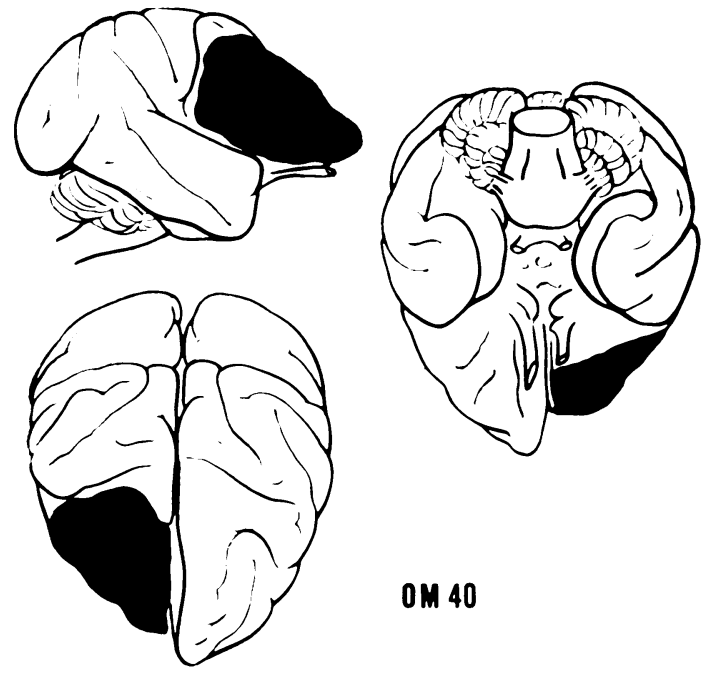

FIG. 1. The site and extent of the cortical ablation (solid black) in the right frontal lobe in experiment $O M 40$. principal nuclei, there is almost total cell loss with accompanying dense gliosis, whereas in the intralaminar nuclei, centralis medialis, paracentralis, and centralis lateralis the change is mainly one of cell shrinkage and pallor together with a moderate degree of gliosis (Fig. 2). (It may be noted that the terminology used for the thalamic nuclei is that of Olszewski (1952); in this atlas the nucleus centralis lateralis is more or less co-extensive, in the anteroposterior dimension, with the centromedian nucleus, and what is referred to as the paracentral nucleus comprises most of the lateral wing of the intralaminar system, excluding what Olszewski termed the nucleus centralis superior lateralis.) Although under low magnification, there appears to be marked cell loss in the posterior parts of these nuclei, a careful examination under higher magnification makes it clear that most of the apparent loss of cells is, in fact, due to the pallor and compacting of the neurones. Without quantitative data it is not possible to assess the degree of the cell loss, but it is our impression that this must be, at the most, slight. In

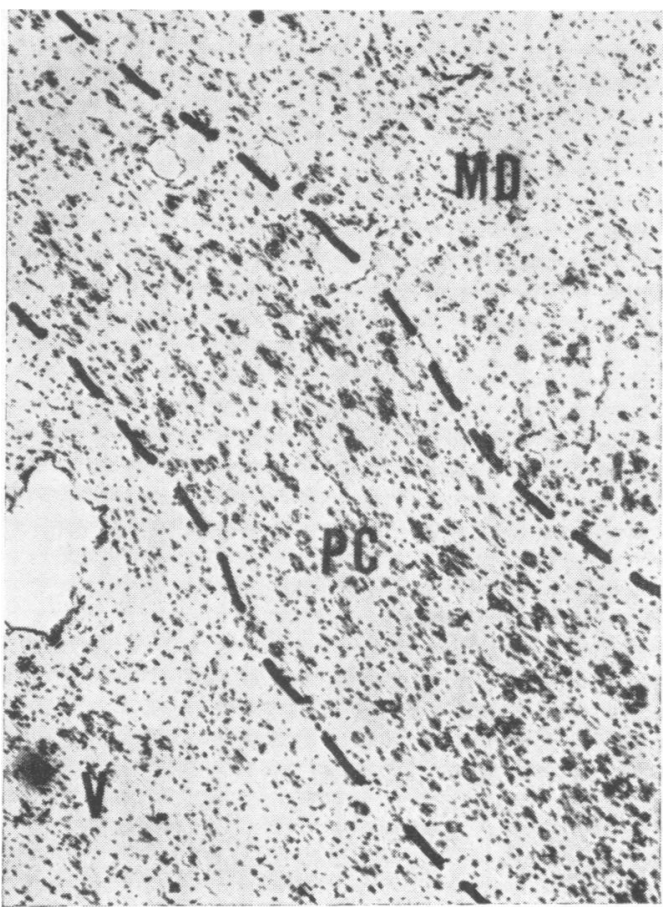

FIG. 2a.

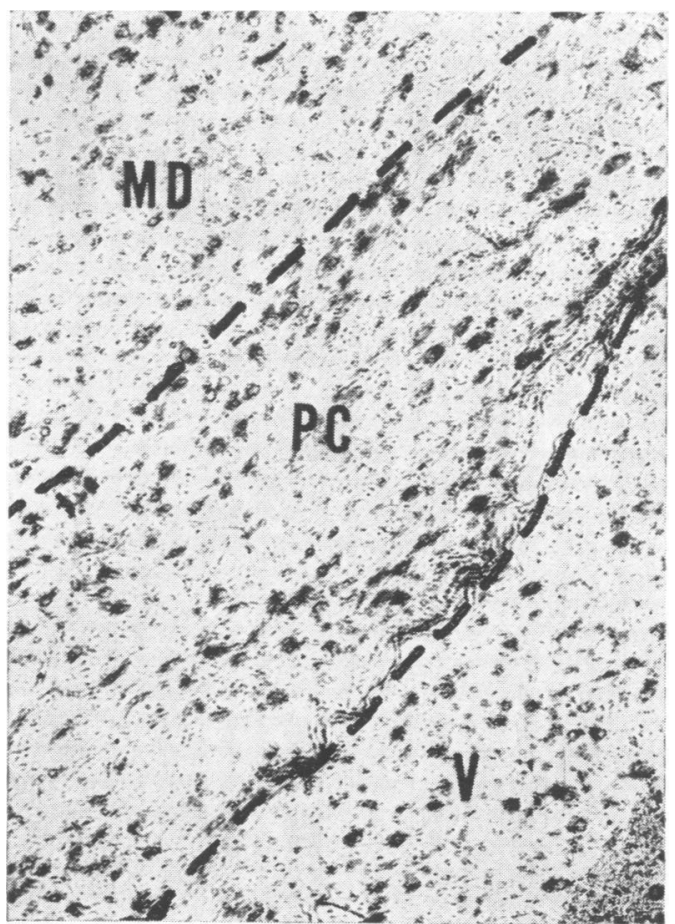

FIG. 2b.

FIG. 2. To show the persistence of shrunken neurones in the nucleus paracentralis and the severe cell loss in the adjoining principal nuclei on the operated side in experiment $O M 40(A)$ as compared with the normal side $(B)$. $\times 88$. $M D$ mediodorsal nucleus. $\quad P C$ nucleus paracentralis. $V$ ventral nucleus. 

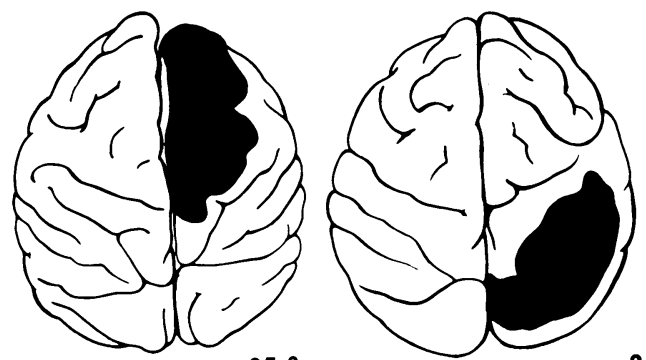

CF 2

OM 45

FIG. 3. The area of cortical damage (solid black) in experiments CF2 and OM45.

both the parafascicular and centromedian nuclei there is quite marked gliosis but no appreciable neuronal degeneration. The preservation of nerve cells in these two nuclei is in marked contrast to the very severe degeneration in the overlying caudal part of the mediodorsal nucleus.

Essentially similar changes have been observed in the intralaminar nuclei in experiment $\mathrm{CF} 2$ after a considerably smaller lesion in the medial part of the dorso-lateral surface of the frontal lobe involving areas $4,6,8,9$, and 10 , and again with no direct involvement of the striatum (Fig. 3). The cellular changes in the intralaminar nuclei, nuclei centralis medialis, paracentralis, and centralis lateralis are again predominantly those of shrinkage and pallor: these changes are most marked in the lateral part of the nucleus paracentralis throughout its anteroposterior extent and in the dorsal part of the nucleus centralis lateralis. The medial part of the nucleus paracentralis and the nucleus centralis medialis shows similar but less marked changes. The ventral part of the nucleus centralis lateralis, the centromedian and parafascicular nuclei show no neuronal degeneration, but there is gliosis in the dorsal parts of the latter two nuclei. One point of interest that may be noted is that in this brain and in OM40 there is an obvious reduction in the crosssectional area of the striatum. This is most marked in the head and anterior part of the body of the caudate nucleus which in OM40 is reduced to about $50 \%$ of the cross-sectional area on the control side.

Not all large lesions of the cerebral cortex result in such degenerative changes in the intralaminar nuclei: in particular, in none of the brains which we have examined with lesions in the parieto-occipital region has there been any sign of cellular degeneration in the rostral intralaminar nuclei. For example, in experiment OM45 there is an area of damage in the parieto-occipital lobe of almost exactly the same size as the lesion in experiment CF2 (see Fig. 3). In the thalamus of this side there are severe degenerative changes in the lateral geniculate nucleus and the pulvinar, but there is no evidence of any cell change or gliosis in the intralaminar nuclei.

The findings in the foregoing experiments have shown that large lesions of the neo-cortex of the frontal lobe cause appreciable shrinkage and pallor of the cells and possibly some degree of cell loss in the intralaminar nuclei, excluding the centromedian and parafascicular nuclei, but lesions of the parietooccipital lobe cause no change. There is, however, a marked difference between the severity of the degeneration in these nuclei after cortical lesions and that seen in the adjoining principal nuclei. In our material it is only when the striatum and internal capsule or the nucleus ventralis anterior of the thalamus are involved that a comparable degree of cell loss occurs in the intralaminar nuclei and main thalamic nuclei. Most of the experiments described in our earlier publication (Powell and Cowan, 1956) were of this type, and a careful re-examination of the material used in that study has confirmed that the intralaminar nuclei do degenerate as severely as the principal nuclei, and that there is a definite correlation between the distribution of the degeneration in these intralaminar nuclei and the site of involvement of the internal capsule. Several additional experiments of this type have been studied in the present series, most of the lesions having resulted from attempts to place a large lesion in the putamen. As the findings in this group are essentially the same as in the previous study, only one case will be described.

In experiment OM17 an electrolytic lesion was placed stereotactically in the putamen. The damage extends from just rostral to the level of the crossing of the anterior commissure back almost to the caudal end of the putamen (Fig. 4). As a result of secondary necrosis due to involvement of the perforating vessels passing through the putamen there is abroad area of damage in the dorsal part of the internal capsule and the caudal part of the head of the caudate nucleus. In the thalamus of this side there is complete cell loss and intense gliosis in the lateral half of the nucleus ventralis anterior, in most of the nucleus ventralis lateralis, in the rostral part of the nucleus ventralis posterior, and in the lateral edge of the posterior part of the mediodorsal nucleus. Anteriorly, all of the intralaminar nuclei are quite intact, but more posteriorly the nucleus centralis lateralis and the dorsal third of the paracentral nucleus show complete cell loss with marked gliosis (Fig. 5). The centromedian nucleus is severely atrophic with virtually complete cell loss and heavy gliosis, but the adjoining parafascicular nucleus is quite intact.

This, and the other experiments in this group, confirm the two main features of the previous study: 

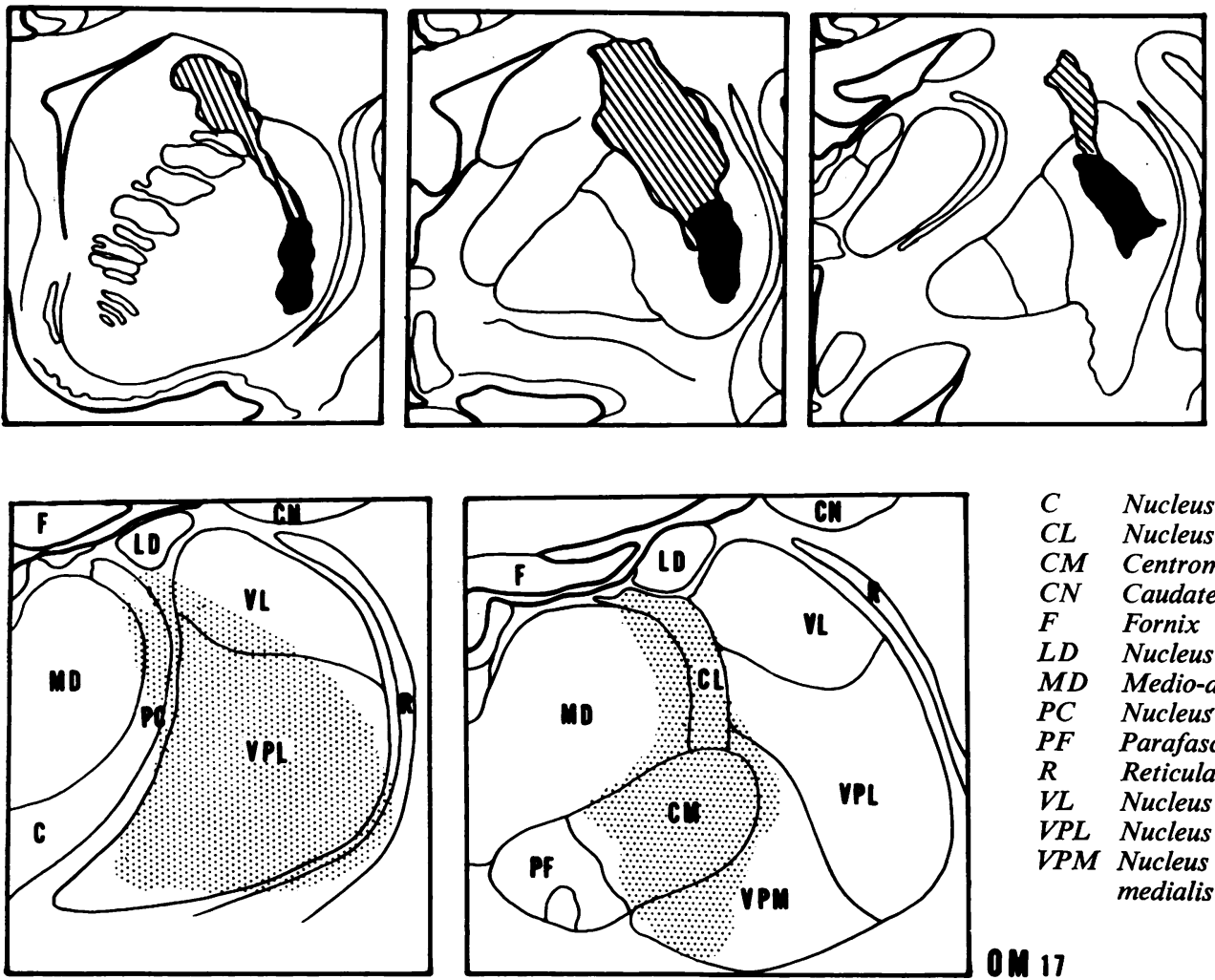

C Nucleus centralis medialis

CL Nucleus centralis lateralis

CM Centromedian nucleus

CN Caudate nucleus

F Fornix

LD Nucleus lateralis dorsalis

MD Medio-dorsal nucleus

$P C \quad$ Nucleus paracentralis

PF Parafascicular nucleus

$R \quad$ Reticular nucleus

$V L \quad$ Nucleus ventralis lateralis

VPL Nucleus ventralis posterior

VPM Nucleus ventralis posterior medialis

\section{OM 17}

FIG. 4. The site of the primary electrolytic lesion (solid black) and the secondary necrosis in the internal capsule and caudate nucleus (cross-hatching) in experiment OM17, together with the resulting cellular degeneration in the intralaminar and adjoining main thalamic nuclei (stippling).
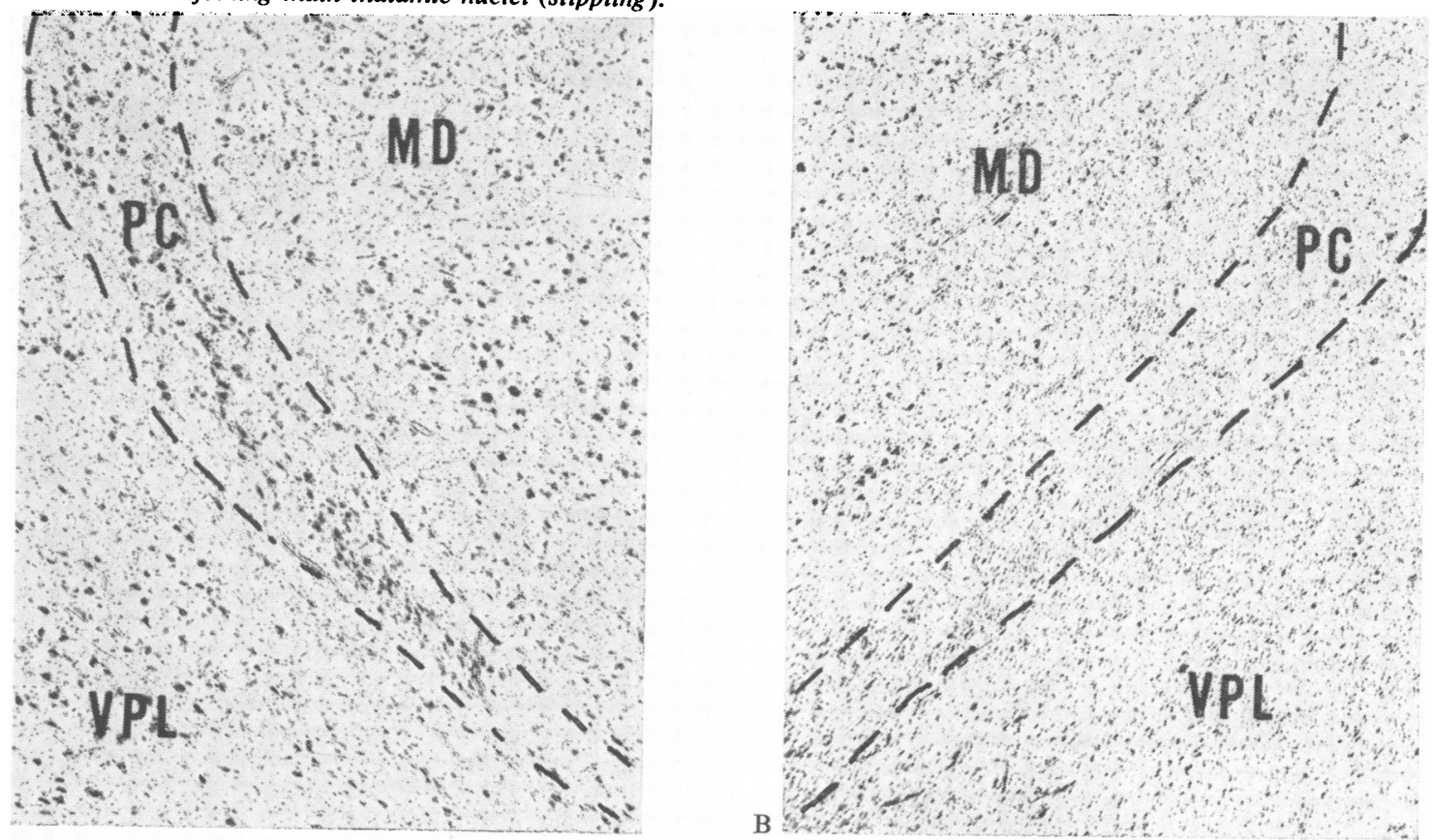

FIG. 5. Photomicrograph showing the severe cell loss and gliosis in the dorsal part of the paracentral nucleus and the adjoining principal nuclei on the operated side in experiment $O M 17(B)$ as compared with the normal side $(A)$. $\times 52$. 
after lesions of the internal capsule there is no difference in severity between the degeneration of the cells of the intralaminar nuclei and those of the main nuclei, and that there is a topographical organization within the projection of these nuclei. As in some of our experiments with cortical damage the lesion extends very close to the caudate nucleus and putamen, it would seem to be impossible to account for the difference in the severity and distribution of the cellular changes in the intralaminar nuclei in these two groups of experiments without implicating the corpus striatum in some way, and the crucial problem in our understanding of the projection of these nuclei is the elucidation of their relationship to the striatum.

In order to determine whether the intralaminar nuclei project upon the caudate nucleus and putamen in a manner comparable to the localized projection of the principal thalamic nuclei upon the neo-cortex, several brains with lesions confined to either the caudate nucleus or the putamen were prepared. These lesions, although individually small, collectively cover the greater part of the cross-sectional area of the head and the anterior part of the body of the caudate nucleus, and almost the whole of the putamen throughout its rostro-caudal extent. It should be emphasized that in none of these experiments has there been any involvement of the internal capsule or of the white matter surrounding the striatum apart from the narrow electrode track or, in some cases, the division of the rostral half of the corpus callosum. As the findings in these experiments are consistent only three brains will be described, two with lesions in the head of the caudate nucleus and the third with a lesion in the putamen.

In experiment OM3 an electrolytic lesion was placed in the caudate nucleus of the right side. Anteriorly, the area of damage is situated centrally in the head of the caudate nucleus, but caudally it occupies the ventromedial part of the head just dorsal to the internal capsule (Fig. 6). Careful and repeated examination of a closely spaced series of sections throughout the thalamus has failed to show any evidence of cellular change in any of the intralaminar nuclei, and in particular in the centralis medialis, paracentralis and centralis lateralis, and there is no sign of gliosis in these nuclei. The principal thalamic nuclei are likewise unaffected. A possible criticism of the findings in this and similar experiments (including those of Nashold et al., 1955) is that in each case the lesion in the caudate nucleus has damaged no more than 10 to $15 \%$ of its total volume. In the next experiment, OM34, a considerable part of the head of the caudate nucleus has been removed on the right side through a transcallosal approach. The lesion in the head of the caudate nucleus extends from its rostral end back to the level of crossing of the anterior commissure, and at its greatest extent has destroyed all but the lateral part of the caudate. Although it approaches close to the internal capsule, at no point does it extend into it (Fig. 6). The only other structures involved are the dorsal part of the septum, the rostral part of the corpus callosum, and a small area of the cingulate cortex. In the thalamus of this side the only degeneration is in the anterior part of the anteromedial nucleus where there is appreciable cell loss and gliosis. The adjoining intralaminar nuclei, centralis medialis and paracentralis are quite free of degeneration, as are the more caudal components of this group, so that it may be said with some confidence that these nuclei do not project exclusively upon the head of the caudate nucleus.

Several attempts have been made to obtain a lesion in the putamen of comparable size to the caudate lesion in OM34, but in each case this has resulted in an area of necrosis extending upwards and medially from the primary electrolytic lesion to involve the dorsal part of the internal capsule and the adjoining caudate nucleus, as in experiment OM17. The necrosis is due to thrombosis of the large perforating vessels which ascend through the putamen to supply more dorsal parts of the striatum and internal capsule (Alexander, 1943), and in our experience it seems unlikely that a substantial parto of the putamen could be destroyed without somed secondary necrosis of adjoining structures. We have however, placed several small lesions in different parts of the putamen and in none of these is there any definite sign of cell loss in the thalamus (and in particular in the centromedian and parafascicular nuclei). The largest lesion of the putamen which has not involved the internal capsule is that in experiment OM9 in which the area of damage is limited to the postero-dorsal part of the putamen, as shown in Figure 6. Apart from the minimal damage in the overlying white matter due to the electrode track, there is no involvement of surrounding structures. In the intralaminar nuclei of the thalamus of this side there is again no cell loss, but in the centromedian nucleus there is slight shrinkage of the cells in the dorso-lateral part of the nucleus. The cellular changes seen in the centromedian nucleus, although slight, are probably significant as similar changes have been found in other parts of the nucleus in experiments with lesions of comparable size in different parts of the putamen.

The results of the three experiments described, and all others of this group, are consistent and it seems reasonable to infer that the intralaminar nuclei do not project in a simple manner upon localized parts of 

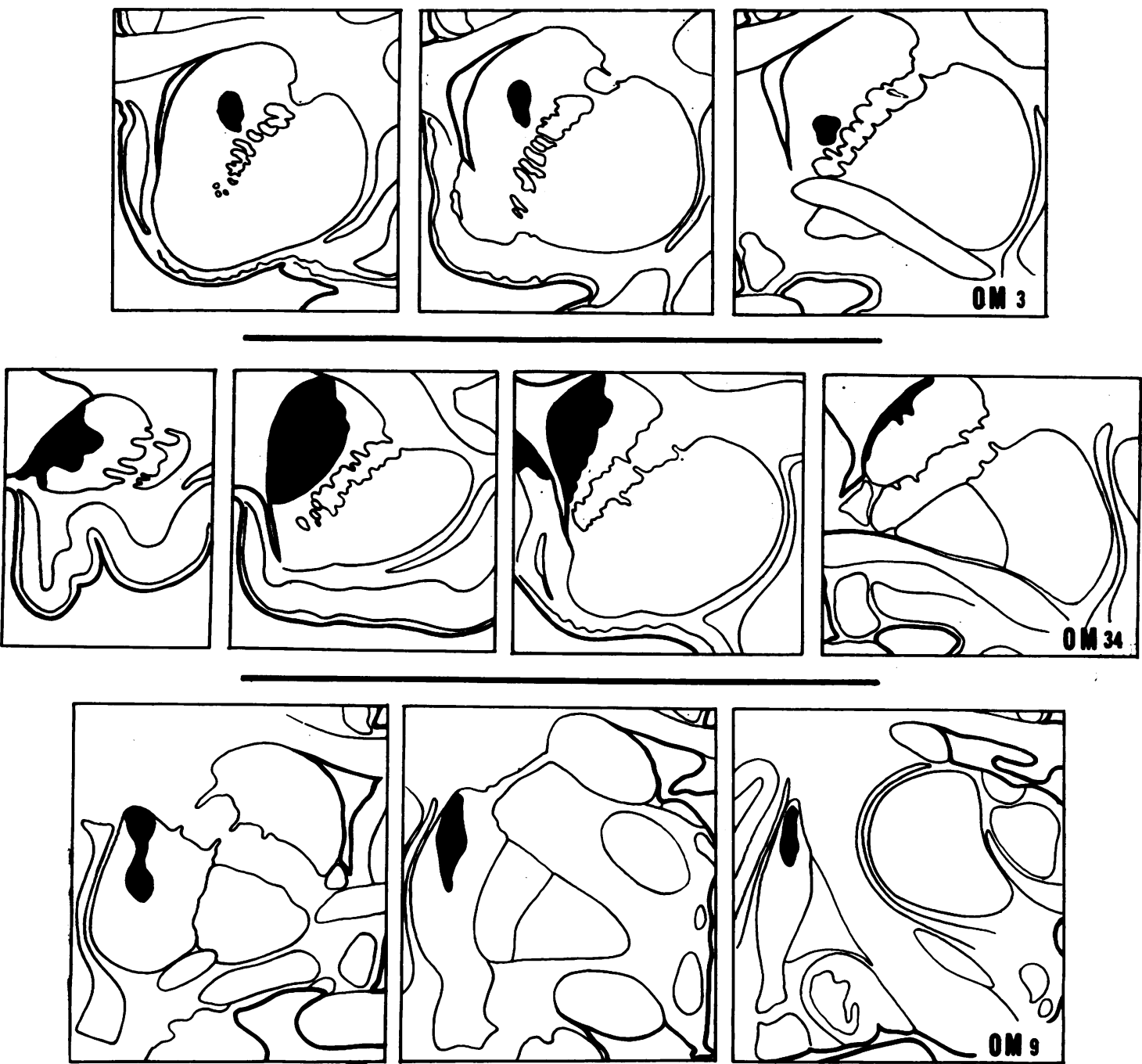

FIG. 6. The sites of the striatal lesions (solid black) in the three experiments OM3, OM34 and OM9. Observe that in none of these is there any involvement of the adjoining white matter.

either the caudate nucleus or of the putamen. The most likely explanations for the absence of cell loss in these nuclei after discrete striatal lesions are either that they project widely upon both parts of the striatum or that, in addition to their striatal connexion, they have a collateral projection to the cerebral cortex. To test the first hypothesis it would be necessary to damage a considerable part of both the putamen and caudate nucleus of one side, without involving the internal capsule, but technically this is extremely difficult to accomplish, and, despite several attempts, we have been unsuccessful. An attempt was made to test the second hypothesis by 5 combining an extensive removal of most of the cortex anterior to the central sulcus with a lesion in the head of the caudate nucleus which had been exposed through the corpus callosum. Examination of the serial sections of this brain (OM39) shows that the extent of the damage to the caudate nucleus was smaller than had been intended, and at its widest part has only destroyed the medial third of the crosssectional area of the head of the caudate. In the thalamus there is severe degeneration in a number of the principal nuclei, including the anterior, medial, and ventral groups, consequent upon the cortical lesion. In the intralaminar nuclei centralis medialis, 
paracentralis and centralis lateralis there is marked shrinkage, pallor, and compacting of the cells, together with some degree of cell loss which is most marked in the nucleus centralis medialis. The centromedian and parafascicular nuclei show gliosis and there is possibly some slight cell shrinkage in the lateral part of the centromedian. These changes are very similar to those seen in experiment OM40 with a lesion in the frontal cortex, and it is impossible to state whether the added striatal involvement in OM39 has resulted in significantly more degeneration, and in particular in a greater degree of cell loss in the intralaminar nuclei, so that unfortunately the experiment must be considered inconclusive.

The severity of the gliosis in all the intralaminar nuclei after cortical lesions appears to be incommensurate with the degree of neuronal atrophy (this is especially true of the centromedian and parafascicular nuclei in which there is little or no cell change). This suggests that the cellular shrinkage with loss of Nissl material seen in these experiments is a reflection of a transneuronal denegeration following the removal of afferents to these nuclei from the cerebral cortex. For this interpretation to be considered correct there should be a substantial projection from the relevant parts of the cortex to the intralaminar nuclei, and in fact such a projection has been demonstrated in several recent studies. The findings in the next group of experiments have confirmed this, and in addition show that in the monkey there is an antero-posterior organization in the projection of the frontal lobe upon the intralaminar nuclei. The animals in this group were allowed to survive for relatively short periods after the operation and the brains were stained for fibre degeneration by the method of Nauta (1957). Only the terminal degeneration in the intralaminar nuclei will be described here, although in each brain extensive fibre degeneration is present in othercortical and subcortical structures.

In the first of these experiments, OM24, most of the prefrontal cortex was removed, including areas 9, 10, 11, 12, and 46 (Fig. 7) of Walker (1940). Following this lesion there is heavy preterminal degeneration in the rostral intralaminar nuclei, centralis medialis and paracentralis, and in the horizontally disposed fibrous lamella above the centromedian and parafascicular nuclei. The latter, however, are quite free of degeneration as is theo overlying nucleus centralis lateralis. In the secon
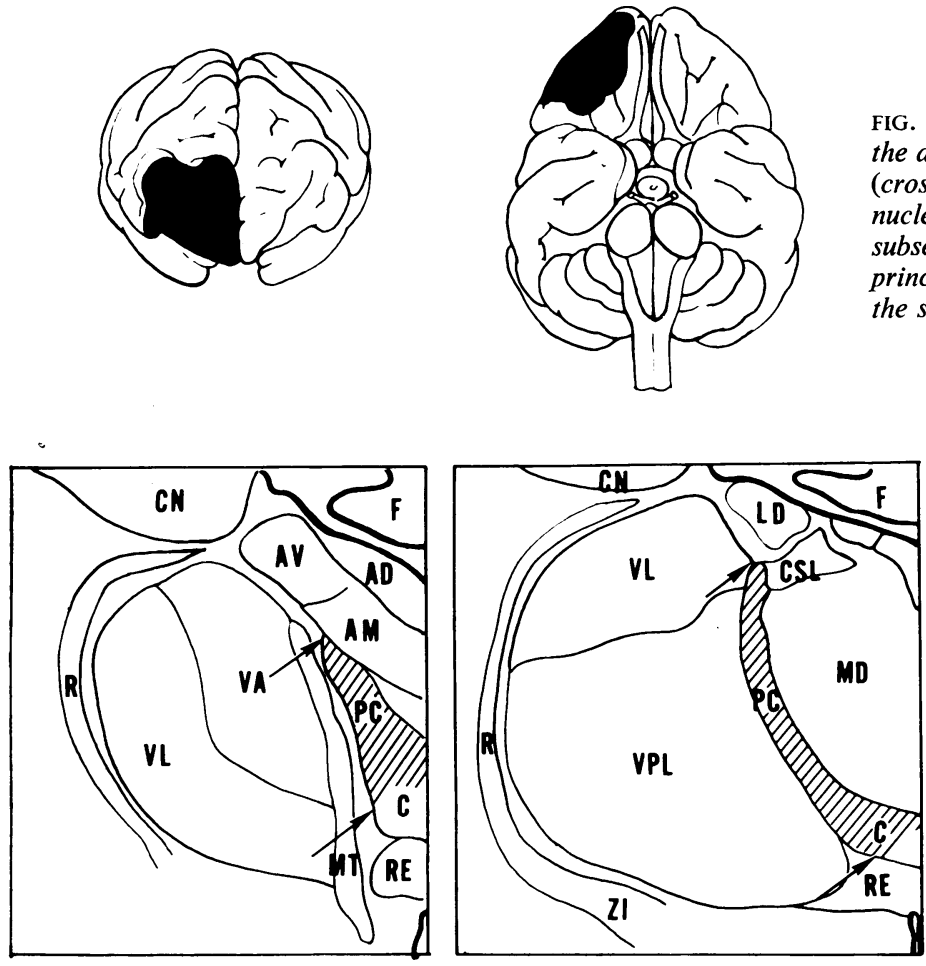

FIG. 7. The cortical lesion (solid black) and the distribution of terminal degeneration (cross-hatching and arrows) in the intralaminar nuclei in experiment OM24. In this and the subsequent figures the fibre degeneration in the principal thalamic nuclei has been omitted for the sake of clarity.

AD Antero-dorsal nucleus

AM Antero-medial nucleus

AV Antero-ventral nucleus

C Nucleus centralis medialis

CN Caudate nucleus

CSL Nucleus centralis superior lateralis

F Fornix

LD Nucleus lateralis dorsalis

MD Medio-dorsal nucleus

MT Mamillo-thalamic tract

$P C \quad$ Nucleus paracentralis

$R \quad$ Reticular nucleus

$R E \quad$ Nucleus reuniens

$V A$ Nucleus ventralis anterior

$V L \quad$ Nucleus ventralis lateralis

VPL Nucleus ventralis posterior lateralis ZI Zona incerta 
brain, OM14, there is a rather smaller lesion in the prefrontal granular cortex (Fig. 8), involving only parts of areas 9,10 , and 12 ; at no point does it encroach upon areas 6 or 8 . In the intralaminar nuclei of this side there is again severe fragmentation in the nucleus paracentralis and the lateral parts of the nucleus centralis medialis, but this degeneration is confined to the rostral sections through these nuclei; their more caudal parts and the nucleus centralis lateralis, and the parafascicular and centromedian nuclei are quite free of degeneration (although there is definite degeneration in the medio-dorsal nucleus at these levels). The findings in these two experiments may be compared with the distribution of the degeneration in the next experiment, OM27, in which there is a large lesion in the motor and premotor areas. In this brain the greater part of the cortex between the central and arcuate sulci (corresponding to areas 4 and 6) was removed (Fig. 9). At rostral levels through the thalamus the intralaminar nuclei show no evidence of fibre degeneration, but immediately in front of the level of the centromedian nucleus a little degeneration is found at about the middle of the medio-lateral extent of the paracentral nucleus. This degeneration increases in severity at more caudal levels and becomes continuous with the very heavy fibre degeneration in the nucleus centralis lateralis. The whole of the centro-

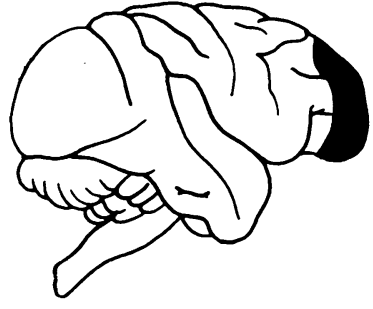

FIG. 8 .

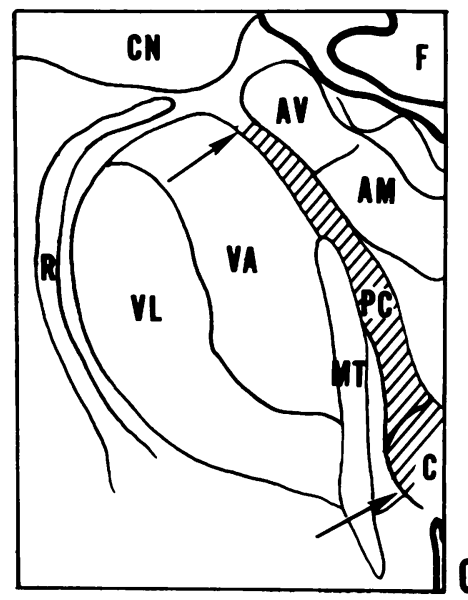

FIG. 8. The lesions (solid black) and terminal degeneration (cross-hatching and arrows) in the rostral intralaminar nuclei in experiment OMI4.

AM Antero-medial nucleus

AV Antero-ventral nucleus

C Nucleus centralis medialis

CN Caudate nucleus

F Fornix

MT Mamillo-thalamic tract

$P C$ Nucleus paracentralis

$R \quad$ Reticular nucleus

$V A$ Nucleus ventralis anterior

$V L \quad$ Nucleus ventralis lateralis

$0 M_{14}$
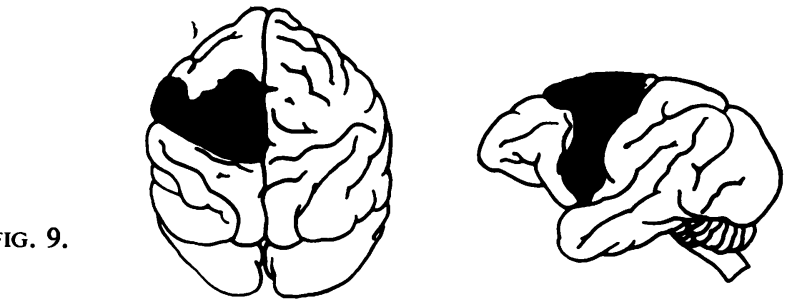

FIG. 9. The extent of the lesion (solid black) and the resultant fibre degeneration in the intralaminar nuclei (cross-hatching and arrows) in experiment OM27.

CL Nucleus centralis lateralis

CM Centromedian nucleus

CSL Nucleus centralis superior lateralis
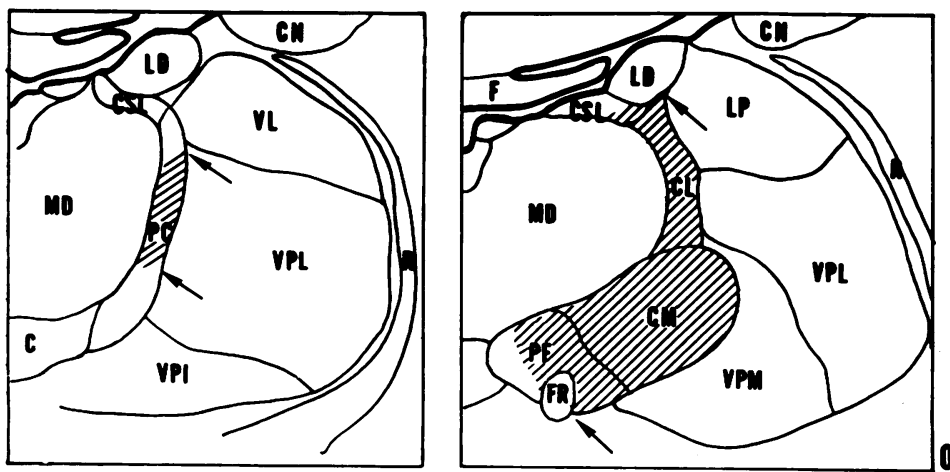

FR Fasisculua retroflexus

LD Nucleus lateralis dorsalis

LP Nucleus lateralis posterior

$M D$ Medio-dorsal nucleus

$P F \quad$ Parafascicular nucleus

VPI Nucleus ventralis posterior inferior

VPL Nucleus ventralis posterior lateralis

VPM Nucleus ventralis posterior medialis 

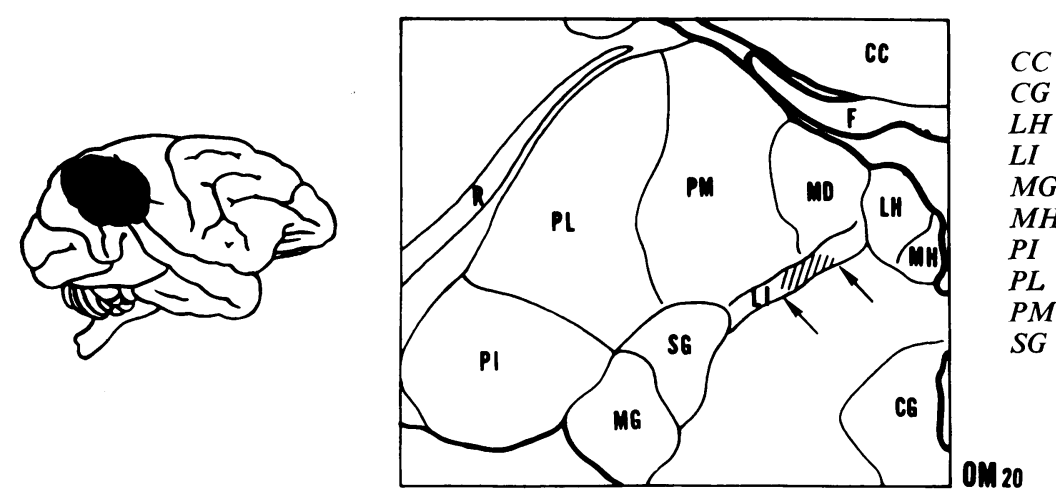

Corpus callosum

$C G$ Central gray

LH Lateral habenular nucleus

LI Nucleus limitans

MG Medial geniculate nucleus

MH Medial habenular nucleus

PI Nucleus pulvarinaris inferior

$P L \quad$ Nucleus pulvarinaris lateralis

$P M \quad$ Nucleus pulvarinaris medialis

$S G \quad$ Suprageniculate nucleus

FIG. 10. The lesion (solid black) in the parieto-occipital region in experiment OM2O and the terminal degeneration in the nucleus limitans (cross-hatching and arrows) in the caudal part of the thalamus.

median nucleus and the lateral half to two-thirds of the parafascicular nucleus are filled with the most severe fibre fragmentation.

The findings in other brains with partial involvement of these areas are consistent with these observations and, in addition to showing an anteroposterior organization in the projection of the cortex of the lateral surface of the frontal lobe upon the intralaminar nuclei, they also indicate that for the centromedian nucleus, at least, there is some degree of medio-lateral organization in the projection from the motor cortex. There is also some evidence that there may be a projection to rostral levels of the intralaminar nuclei from the cingular cortex, but our material is inconclusive on this point.

In brains with lesions of varying size in the parietal and occipital lobes, on the other hand (such as that shown in Fig. 10), axonal degeneration is not found in the nuclei centralis medialis, paracentralis and centralis lateralis, nor in the centromedian and parafascicular nuclei. In this experiment (OM20) there is, however, some preterminal degeneration in the nucleus limitans close to its posterior end.

Another possible explanation for the degenerative changes in the cells of the intralaminar nuclei is that these are 'retrograde transneuronal' in nature, i.e., they are secondary to the severe retrograde atrophy in the principal thalamic nuclei. This interpretation is based upon the possibility that the cells of the intralaminar nuclei may send axon collaterals into the adjoining main nuclei, and if this is so it might reasonably be expected that some evidence of cellular atrophy would be seen in the intralaminar nuclei after localized lesions in the principal nuclear groups. No systematic attempt has been made to investigate this possibility, but the findings in a few experiments with stereotactic lesions in the thalamus will ke briefly described. In two of these experiments,
OM33 and MA5, the lesions have destroyed the $\overrightarrow{\vec{\omega}}$ greater part of the anterior nuclear group, the entire nucleus lateralis dorsalis and the dorsal half of the medio-dorsal nucleus. In neither of these cases is there any suggestion of cellular change in the intralaminar nuclei. On the other hand, lesions in the nucleus ventralis anterior, as in experiment OM1 consistently result in severe degeneration in th intralaminar nuclei: this is adequately accounted for by the fact that the extrathalamic projection from these nuclei passes rostrally through this componerf of the ventral nuclear group. The distribution of the degeneration in these experiments with lesions in the nucleus ventralis anterior varies according to the siet. of injury in the nucleus, which again confirms that there is an organization in the efferent projection pathway of the intralaminar nuclei.

\section{DISCUSSION}

Despite the variety of experiments which have been carried out in this and other studies, it is still not possible to provide a definite answer to the problem of the precise site or mode of termination of the efferent fibres of the intralaminar nuclei of the $\overline{3}$ thalamus. To a considerable extent this must be? attributed to the relative insensitivity of cellular degeneration techniques and to the wide range of possible reactions that cells may undergo either after $\frac{1}{3}$ axon section or after deprivation of their afferents.

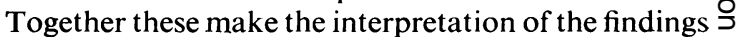
of experiments based solely upon cellular methods $\frac{D}{O}$ extremely difficult, as is being appreciated more and more. In the absence of supportive evidence from the $N$ use of other techniques it may be almost impossible to provide a satisfactory explanation for either the presence or the absence of cellular change after an $\omega$ experimental lesion; cells may show no change after? 
indubitable interruption of their axons (Daitz and Powell, 1954) or they may undergo profound atrophy and even death after suffering no direct injury but simply as the result of deafferentation (c.f. Matthews, Cowan, and Powell, 1960) or secondary to the death of cells upon which they terminate (Cowan and Powell, 1954). The interpretation of the degenerative changes in the cells of the intralaminar nuclei after various cortical and subcortical lesions exemplifies these difficulties as will become apparent in the ensuing discussion. But, unfortunately, this is the only experimental method available at present as it is clearly not possible to define the projection of these nuclei (with the exception of the centromedian nucleus) with fibre degeneration methods; as the nuclei are only a few cells wide and are curved in the dorso-ventral and antero-posterior dimensions, it is well-nigh impossible to place a lesion confined within their limits, and, even if this could be achieved, such a lesion would inevitably interrupt large numbers of fibres passing forwards and laterally from the adjoining main nuclei. In view of this there is no alternative but to study the cellular changes in the intralaminar nuclei after different lesions in the cerebral hemisphere and to endeavour to interpret them in the light of ancillary evidence based upon other techniques.

That some degree of cellular degeneration may occur in the intralaminar nuclei after large lesions of the cerebral cortex has been noted by several authors (Walker, 1938; Nashold et al., 1955; Peacock and Combs, 1965; Murray, 1966), and the observations in the first group of experiments of the present series are in agreement with this. Before attempting to interpret the significance of this finding it is necessary to discuss certain features of the degenerative change. Perhaps the first and most important is that the changes in the rostral intralaminar nuclei after cortical lesions are primarily in the form of shrinkage and reduction of Nissl material but with little or no cell loss. As we have pointed out, without recourse to quantitative studies it is impossible to assess the precise degree of cell loss in sites in which there is appreciable cell shrinkage and gliosis. In this context it is interesting to note that Matthews (1964) found a cell loss of only 15 to $20 \%$ in the deafferentated laminae of the monkey lateral geniculate nucleus which were 'so severely atrophied as to be inconspicuous when viewed at low magnifications', and from a personal examination of the brains used by Matthews it may be said that the affected laminae of the lateral geniculate nucleus appear to be much more severely atrophied than are the intralaminar nuclei after cortical lesions. A second point to be stressed is that these changes in the intralaminar nuclei are only found after fairly extensive cortical ablations. Thirdly, in our material cell atrophy was observed in the intralaminar nuclei centralis medialis, paracentralis and centralis lateralis only after lesions of the frontal lobe, and no change was detectable after lesions of comparable size in the parieto-occipital region. Murray (1966), in a study of this problem in the cat, has adduced evidence for a topographical relationship between the intralaminar nuclei and the cortex 'since rostral sectors of the central medial and paracentral nuclei show cell loss after lesions involving anterior limbic and orbito-frontal cortex while caudal sectors degenerate after lesions involving cingular and parieto-occipital cortex'. The available evidence from studies in the Primate brain are consistent with such an arrangement for the nuclei centralis medialis and paracentralis; the nucleus centralis lateralis, however, shows no sign of cellular degeneration after lesions of the parietooccipital areas. Fourthly, there is general agreement that the centromedian and parafascicular nuclei show no significant neuronal atrophy even after hemidecortication (Walker, 1938; Nashold et al., 1955; Peacock and Combs, 1965).

Several explanations may be put forward to account for the cellular changes in the rostral intralaminar nuclei after cortical lesions. The first is that they may be retrograde in nature subsequent to involvement of the intralaminar axon terminals in the cortex. If this is so, however, the relationship of the intralaminar nuclei to the cortex must differ significantly from that of the principal thalamic nuclei since the degeneration in the two systems after cortical lesions is quite different; whereas the principal nuclei undergo almost total cell loss after lesions restricted to one or other cortical area, the majority of the cells of the intralaminar nuclei persist even after extensive cortical lesions. This difference cannot simply be explained on the basis of an unusually widespread cortical projection from these nuclei as the changes do not appear to be more severe after total removal of the cerebral cortex (Nashold et al., 1955; Peacock and Combs, 1965). Since after lesions of the internal capsule the intralaminar nuclei undergo total cell degeneration, exactly comparable in appearance and time course with that seen in the principal thalamic nuclei, it may reasonably be inferred that the projection of these nuclei cannot be exclusively to the cerebral cortex. The possibility remains, however, that the projection fibres from these nuclei give off collaterals to the cortex; in an earlier study (Powell and Cowan, 1956) we tended to discount this possibility because we had not observed cellular atrophy in the intralaminar nuclei after the small cortical lesions 
studied at that time, and, as we pointed out in that paper, there is no good evidence, either anatomical or physiological, for such collaterals. The widely held view that the so-called non-specific cortical afferents of Lorente de Nó (1943) have their origin specifically in the intralaminar nuclei is not, as far as we are aware, based upon direct evidence.

As we have suggested in the results, an alternative explanation for the cellular changes in the intralaminar nuclei after cortical lesions is that these are transneuronal following the removal of cortical afferents to these nuclei (i.e., transneuronal in the classical use of this term for the cellular atrophy found in the lateral geniculate nucleus after section of the optic nerve). This possibility was considered by Nashold $e t$ al. (1955), but they were inclined to dismiss it on the grounds that the changes in the intralaminar nuclei were too advanced for a postoperative survival period of only two months. This seems to have been due to a misinterpretation of the findings of Cook, Walker, and Barr(1951) on the timecourse of transneuronal degeneration in the lateral geniculate nucleus of the cat and rabbit. In fact, in the cat transneuronal changes are maximal within two months of deafferentation both in the lateral geniculate nucleus and in certain of the auditory relay nuclei (Powell and Erulkar, 1962), and in Primates marked changes are apparent in the lateral geniculate nucleus within a few days after eye enucleation (Matthews etal., 1960). This view deserves reappraisal, especially since it has become clear that transneuronal degeneration may be of widespread occurrence, having been demonstrated in relay nuclei of most of the sensory systems, in several nuclei of the brain-stem, and in sympathetic ganglia (see Cook et al., 1951; Hamlyn, 1954; Torvik, 1956; Matthews et al., 1960; Matthews and Powell, 1962; Powell and Erulkar, 1962; Peacock and Combs, 1965). Transneuronal effects must therefore be seriously considered as a possible explanation, or at least as a contributory factor, in all cases of neuronal degeneration when there is evidence that the causative lesion may have involved an appreciable number of afferents to the affected area, and especially where the distribution of the cellular degeneration agrees with the topical organization of the afferents. For these reasons it has been suggested that the cellular changes which are seen in the thalamic reticular nucleus after cortical lesions are transneuronal in nature (Carman, Cowan, and Powell, 1964a), and a similar explanation could account for the rather similar changes found in the claustrum (c.f. Narkiewicz, 1964; Carman, Cowan, and Powell, 1964b). In the case of the intralaminar nuclei all the necessary conditions for transneuronal degeneration are present. First, the nature of the cellular change is entirely consistent with that described in sites where the degeneration is un- $Z$ questionably transneuronal; secondly, there is now good evidence from a number of studies for a substantial projection from the cerebral cortex to these nuclei (Auer, 1956; Niimi, Kishi, Miki, and

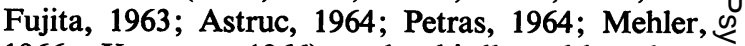
1966; Kuypers, 1966) and, thirdly, although a $\frac{\widehat{S}}{\zeta}$ systematic study of the organization of the corticointralaminar projection has not yet been reported, the available evidence suggests that the arrangement $\vec{\Rightarrow}$ of the cortical afferents to the intralaminar nuclei $\stackrel{9}{+}$ is in agreement with the distribution of the cellular changes in these nuclei after cortical lesions.

A third possible explanation for the cellular $\frac{\bar{D}}{\bar{D}}$ degeneration in the intralaminar nuclei which must $\stackrel{\mathbb{Q}}{\varrho}$

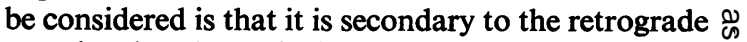
atrophy in the principal thalamic nuclei. Such $\overrightarrow{0}$ retrograde transneuronal degeneration is known to occur in at least two sites; in the medial mamillary $\vec{\omega}$ nucleus after damage to the cingulate cortex (Cowan and Powell, 1954) and in the retina after lesions of the visual cortex (Walsh, 1947; Haddock and Berlin, 1950; Van Buren, 1963), and has been considered as a possible cause of the degeneration in the thalamis ? reticular nucleus (Rose, 1952). This explanatio $\vec{m} \vec{t}$ refers, of course, only to the changes found in the intralaminar nuclei after lesions of the cerebraif cortex and cannot be regarded as the main causative $\rightarrow$ factor underlying the very severe changes found aftes lesions of the internal capsule. Perhaps the stronges factor favouring this interpretation is the fact tha $\frac{1}{7} \cdot \vec{\circ}$ the changes in the intralaminar nuclei are always. accompanied by severe atrophy in the adjoining main nuclei. On the other hand, partial direct destruction of certain of the main nuclei (excluding the nucleus ventralis anterior) does not result in appreciable change in the cells of the intralaminar system. (As we have pointed out above, the degeneration in the intralaminar nuclei after involvement of the nucleus ventralis anterior is adequately accounted for by the interruption of the extrathalamic projection fibres of these nuclei.)

In summary, it may be said that although it is not possible at present to decide which of these interpretations is correct, in our judgment the balance of the evidence which is available favours the view that the changes in the intralaminar nuclei after cortical lesions are transneuronal in nature; certainly this is the only explanation for which all the necessary conditions have been satisfied. The only observation which is difficult to account for if this hypothesis is correct is the absence of cellular degeneration in the centromedian and parafascicular nuclei after hemidecortication, even though these are known to receive a heavy projection from the motor cortex. 
This difference in the reaction of the centromedian and parafascicular nuclei to cortical injury must imply that they differ in some basic way from the other intralaminar nuclei, but the nature of this difference remains to be determined. It should be emphasized that whatever factor may be responsible for the difference in their reaction to cortical damage, all of the intralaminar nuclei degenerate completely after a lesion of the internal capsule and striatum. This suggests that the relationship of all the intralaminar nuclei to the striatum, at least, is probably the same, as we shall discuss more fully later. It is of interest in this context to note that these two groups of intralaminar nuclei are known to differ in their afferent connexions, the rostral intralaminar nuclei receiving a projection from the deep nuclei of the cerebellum, whereas the centromedian nucleus, at least, is in receipt of fibres from the globus pallidus.

The dramatic difference in the degeneration in the intralaminar nuclei, both in degree and distribution, following a lesion of the internal capsule at the level of the striatum and after extensive cortical removals which spare the striatum compels one to conclude that the striatum is, in some way, implicated in the projection of the intralaminar nuclei. The previous findings that the distribution of the degeneration in the intralaminar nuclei varies in an organized manner with the site of the lesion in the internal capsule and striatum naturally led to the suggestion that these nuclei project in a localized manner upon the caudate nucleus and the putamen (Droogleever Fortuyn and Stefens, 1951; Stefens and Droogleever Fortuyn, 1953; Powell and Cowan, 1954, 1956; Cowan and Powell, 1955). However, Nashold et al. (1955) have shown that small lesions restricted to the caudate nucleus or the putamen result in no cellular degeneration in the thalamus, and the present experiments have confirmed these fundings, and in addition have demonstrated that considerably larger lesions of the caudate nucleus, which do not involve the internal capsule, also cause no degeneration in the intralaminar nuclei. On the other hand, in a recent study using the Nauta method, Mehler (1966) has shown that following a lesion placed stereotactically in the centromedian nucleus of the monkey axonal degeneration can be traced forwards through the nucleus ventralis anterior and the reticular nucleus, across the internal capsule, to a wide termination in the putamen and body of the caudate nucleus. Although in this experiment there was some evidence of terminal degeneration in the reticular nucleus, there was apparently no projection to the cerebral cortex; the little degeneration that was seen in the cortex seeming to be incidental to the passage of the electrode. Bowsher (1966), however, claims that following lesions in the centromedian nucleus of the cat degenerating fibres pass up to the cortex of the anterior marginal and posterior suprasylvian gyri, but no preterminal degeneration was seen within the cortex itself, and after a similar lesion in one cynomologous monkey 'a rather small but significant amount of preterminal degeneration' was seen in the deepest layers of the cortex in the region of the superior frontal gyrus.

The absence of cellular degeneration in the intralaminar nuclei after lesions restricted to the caudate nucleus and putamen may be interpreted in two different ways. First, it is possible that the cells of the intralaminar nuclei project in a localized manner upon the corpus striatum but also send collaterals to the cortex, and that the latter serve to maintain the integrity of the neurones after striatal lesions. Our attempt to test this possibility experimentally by combining a striatal lesion with ablation of a large part of frontal lobe was unfortunately inconclusive. The fact that Mehler (1966) could find no axonal degeneration in the cerebral cortex after a lesion in the centromedian nucleus must therefore be considered conclusive evidence for this nucleus, at least. It may not be justifiable to extend it to the rest of the intralaminar nuclei; first, because the cells of the rostral intralaminar nuclei react quite differently to cortical injury than those of the centromedian nucleus and, secondly, while the rostral intralaminar nuclei show no change after localized lesions in the striatum there is often a suggestion of cell shrinkage in the centromedian nucleus after lesions confined to the putamen.

The second interpretation is that each of the intralaminar nuclei projects widely upon the striatum and possibly to both the caudate nucleus and the putamen (c.f. Gerebtzoff, 1940; Vogt and Vogt, 1941 ; Freeman and Watts, 1947; McLardy, 1948; Hassler, 1949; Simma, 1951). As most of the evidence which has been cited in support of this view is based upon human pathological material and concomitant involvement of the internal capsule cannot be excluded, considerable weight must be attached to Mehler's observation (1966) that the efferents from the centromedian nucleus terminate widely in both the putamen and body of the caudate nucleus. For the reasons given earlier, it is unfortunately not possible to obtain direct evidence of this kind for the rostral intralaminar nuclei. If it could be shown that the rostral intralaminar nuclei do project widely upon the striatum in the same manner as the centromedian nucleus it would mean that the thalamo-striate projection is organized in the same manner as the cortico-striate system; although the details of the cortico-striate projection 
have not been studied in the monkey, from what evidence is available it would appear that a considerable part of the cortex projects in an organized manner upon both the caudate nucleus and the putamen as has been shown in the rabbit (Carman, Cowan, and Powell, 1963).

It may be helpful at this stage to state that, in our opinion, the most satisfactory interpretation of the available evidence is that the intralaminar nuclei project widely, but in an organized manner, upon both parts of the striatum (the rostral intralaminar nuclei projecting upon the head of the caudate nucleus and the adjoining rostral part of the putamen, and the centromedian and parafascicular nuclei to the body of the caudate and the more caudal part of the putamen), and that the degenerative changes seen in certain of these nuclei after cortical lesions is transneuronal in nature. However, the possible involvement of collateral connexions to the cortex as a cause of the cellular changes in these nuclei after cortical lesions cannot be excluded, but direct evidence for such collaterals is still lacking.

If the conclusion that the intralaminar nuclei project predominantly, if not exclusively, upon the striatum is correct it is of interest to note that both these nuclei and the striatum receive a substantial and apparently well-organized projection from the cerebral cortex. Furthermore, there is some evidence that certain areas of the cortex, at least, may influence particular elements of the intralaminar system and those parts of the striatum to which these particular nuclei project. The significance of the organized cortico-striate projection has been dealt with elsewhere (Carman et al., 1963), and here only two points about the cortical projection upon the intralaminar nuclei will be considered. First, there is the question as to whether the organization of this projection is simply on a topographical basis, as seems to be true of the cortico-striate projection or whether functionally different areas of the cortex project upon different elements of the intralaminar complex. The available evidence is compatible with either of these views, but the fact that the centromedian nucleus receives its cortical fibres from a cytoarchitecturally distinct region, area 4 , argues in favour of the latter. In this context it is of interest to note that in one brain with a lesion of the motor cortex (OM27) terminal degeneration was found in those parts of the caudate nucleus and putamen which Mehler (1966) has shown to receive afferents from the centromedian nucleus. This close relationship of the centromedian nucleus to the motor cortex may well provide a clue both to the remarkable development of this nucleus in the Primate brain and also perhaps to its functional significance. Secondly, there is the problem of the relation of the cortex behind the central sulcus to the intralaminar nuclei. If the apparent topographical organization of the projection of the cortex upon the intralaminar nuclei is continued caudally it might be predicted that the parieto-occipital region would send fibres to the most caudal intralaminar nuclei, the suprageniculate nucleus and the nucleus limitans. These nuclei have usually been regarded as components of the intralaminar system, on both anatomical (Walker, 1938) and physiological (Jasper, 1960) grounds, but recently have been included, with others, in the posterior group (Rose and Woolsey, 1958; Poggio and Mountcastle, 1960). In the cat, at least, it has been shown that parts of the posterior group receive fibres from the posterior and lateral areas of the cortex (unpublished observations), and in the brain of one monkey of the present series, OM20 with a lesion in the parieto-occipital region, there is some axonal degeneration in the nucleus limitans. It is also not without significance that the cellular changes which these nuclei (and the posterior group as a whole) undergo after large lesions of the cortex of the posterior half of the hemisphere in the cat appear to be identical to those found in the rostral intralaminar nuclei, and that in the rat theres is experimental evidence for a projection of the suprageniculate nucleus to the caudal part of then putamen (Droogleever Fortuyn and Minderhoud 1965).

\section{SUMMARY}

An attempt has been made to determine the cause of the cellular changes in the intralaminar nuclei of the thalamus after cerebral lesions. A variety of experiments have been performed in a series of macaque monkeys; these include lesions of the cerebral cortex, internal capsule, caudate nucleus, and putamen, and of certain of the thalamic nuclei. The rostral intralaminar nuclei show cell shrinkage and pallor, but little or no cell loss, after lesions of the frontal lobe; complete cellular degeneration of these nuclei has been found only after involvement of the striatum and the internal capsule. After lesions confined to the caudate nucleus (without encroachment upon the internal capsule) no degeneration is found in the intralaminar nuclei; after localized lesions of the putamen slight cell shrinkage may be seen in the centromedian nucleus. There is a substantial and topographically organized projection of fibres from the cortex of the frontal lobe to the intralaminar nuclei.

From these observations and other recent evidence it is concluded that each of the intralaminar nuclei projects widely, but in an organized manner, upon both parts of the striatum. The presence of axon 
collaterals to the cortex cannot be excluded, but the cellular changes in the intralaminar nuclei after cortical lesions may be adequately accounted for by transneuronal effects following the removal of cortical afferents to these nuclei.

This work was supported by a grant from the Medical Research Council.

\section{REFERENCES}

Alexander, L. (1942). The vascular supply of the strio-pallidum. Res. Publ. Ass. nerv. ment. Dis., 21, 77-132.

Astruc, J. (1964). Corticofugal fiber degeneration following lesions of area 8 (frontal eye field) in Macaca mulatta. Anat. Rec., 148, 256.

Auer, J. (1956). Terminal degeneration in the diencephalon after ablation of frontal cortex in the cat. J. Anat. (Lond.), 90, 30-41.

Bowsher, D. (1966). Some afferent and efferent connections of the parafascicular-center median complex. In The Thalamus, edited by D. P. Purpura and M. D., Yahr, pp. 99-108. Columbia University Press, New York.

Carman, J. B., Cowan, W. M., and Powell, T. P. S. (1963). The organization of cortico-striate connexions in the rabbit. Brain, 86, 525-562.

- $, \ldots,-1$ (1964a). Cortical connexions of the thalamic reticular nucleus. J. Anat. (Lond.), 98, 587-598.

_ - (1964b). The cortical projection upon the claustrum. J. Neurol. Neurosurg. Psychiat., 27, 46-51.

Cook, W H., Walker, J. H., and Barr, M. L. (1951). A cytological study of transneuronal atrophy in the cat and rabbit. J. comp. Neurol., 94, 267-291.

Cowan, W. M., and Powell, T. P. S. (1954). An experimental study of the relation between the medial mamillary nucleus and the cingulate cortex. Proc. roy. Soc. B., 143, 114-125.

-_- _- (1955). The projection of the midline and intralaminar nuclei of the thalamus of the rabbit. J. Neurol. Neurosurg. Psychiat., 18, 276-279.

Daitz, H. M., and Powell, T. P. S. (1954). Studies of the connexions of the fornix system. Ibid., 17, 75-82.

Droogleever Fortuyn, J., and Minderhoud, J. M. (1965). Efferent connections of the supra-geniculate nucleus in the albino rat. J. comp. Neurol., 124, 203-213.

- - and Stefens, R. (1951). On the anatomical relations of the intralaminar and midline cells of the thalamus. Electroenceph. clin. Neurophysiol., 3, 393-400.

Freeman, W., and Watts, J. W. (1947). Retrograde degeneration of the thalamus following prefrontal lobotomy. J. comp. Neurol., 86, 65-93.

Gerebtzoff, M. A. (1940). Les connexions thalamo-striées. Le noyau parafasciculaire et le centre médian. J. belge Neurol., Psychiat., 40, 407-416.

Haddock, J. N., and Berlin, L. (1950). Transsynaptic degeneration in the visual system. Arch. Neurol. Psychiat. (Chic.), 64, 66-73.

Hamlyn, L. H. (1954). The effect of preganglionic section on the neurons of the superior cervical ganglion in rabbits. J. Anat. (Lond.), 88, 184-191.

Hassler, R. (1959). Uber die afferente Leitung und Steuerung des striaren Systems. Nervenarzt, 20, 537-541.

Jasper, H. H. (1960). Unspecific thalamocortical relations. In Handbook of Physiology, Section I: Neurophysiology, Vol. 2, pp. 1307-1321. American Physiological Society, Washington, D.C.

Kuypers, H. G. J. M. (1966). Discussion. In The Thalamus, edited by D. P. Purpura and M. D. Yahr, pp. 122-126, Columbia University Press, New York.

Lorente de Nó, R. (1943). In Physiology of the Nervous System by J. F. Fulton, 2nd ed., pp. 274-301. Oxford University Press, London.
Matthews, M. R. (1964). Further observations on transneuronal degeneration in the lateral geniculate nucleus of the macaque monkey. J. Anat. (Lond.), 98, 255-263.

- Cowan, W. M., and Powell, T. P. S. (1960). Transneuronal cell degeneration in the lateral geniculate nucleus of the macaque monkey. Ibid., 94, 145-169.

cell degeneration in the olfactory bulb of the rabbit. Ibid., 96, 89-102.

McLardy, T. (1948). Projection of the centromedian nucleus of the human thalamus. Brain, 71, 290-303.

Mehler, W. R. (1966). Further notes on the center median nucleus of Luys. In The Thalamus, edited by D. P. Purpura and M. D. Yahr, pp. 109-127. Columbia University Press, New York.

Murray, M. (1966). Degeneration of some intralaminar nuclei after cortical removals in the cat. J. comp. Neurol., 127, 341-368.

Narkiewicz, O. (1964). Degenerations in the claustrum after regional neocortical ablations in the cat. J. comp. Neurol., 123, 335-355.

Nashold, B. S., Hanbery, J., and Olszewski, J. (1955). Observations on the diffuse thalamic projections. Electroenceph. clin. Neurophysiol., 7, 609-620.

Nauta, W. J. H. (1957). In New Research Techniques of Neuroanatomy, edited by W. F. Windle, pp. 17-26. Charles C. Thomas, Springfield, Illinois.

Niimi, K., Kishi, S., Miki, M., and Fujita, S. (1963). An experimental study of the course and termination of the projection fiber from cortical areas 4 and 6 in the cat. Folio psychiat. neurol. jap., 17, $167-216$.

Olszewski, J. (1952). The Thalamus of the Macaca Mulatta. Karger, Basel.

Peacock, J. H., and Combs, C. M. (1965). Retrograde cell degeneration in diencephalic and other structures after hemidecortication of rhesus monkeys. Exp. Neurol., 11, 367-399.

Petras, J. M. (1964). Some fiber connections of the precentral cortex (areas 4 and 6) with the diencephalon in the monkey (Macaca mulatta). Anat. Rec., 148, 322.

Poggio, G. F., and Mountcastle, V. B. (1960). A study of the functional contributions of the lemniscal and spinothalamic systems to somatic sensibility. Johns Hopk. Hosp. Bull., 106, 266-316.

Powell, T. P. S., and Cowan, W. M. (1954). The connexions of the midline and intralaminar nuclei of the thalamus of the rat. J. Anat. (Lond.), 88, 307-319.

(1956). A study of thalamo-striate connexions in the monkey. Brain, 79, 364-390.

- , and Erulkar, S. D. (1962). Transneuronal cell degeneration in the auditory relay nuclei of the cat. J. Anat. (Lond.), 96, 249-268.

Rose, J. E. (1952). The cortical connexions of the reticular complex of the thalamus. Res. Publ. Ass. nerv. ment. Dis., 30, 454-479.

-, and Woolsey, C. N. (1958). Cortical connections and functional organization of the thalamic auditory system of the cat. In Biological and Biochemical Bases of Behavior, edited by H. F. Harlow and C. N. Woolsey, pp. 127-150. University of Wisconsin Press, Madison.

Simma, K. (1951). Zur Projektion des Centrum medianum und Nucleus parafascicularis thalami beim Menschen. Mschr. Psychiat. Neurol., 122, 32-46.

Stefens, R., and Droogleever Fortuyn, J. (1953). Contribution à l'étude de la structure et de quelques connexions des noyaux. intermédiaires du thalamus chez le lapin. Schweiz. Arch. Neurol. Psychiat., 72, 299-318. .

Torvik, A. (1956). Transneuronal changes in the inferior olive and pontine nuclei in kittens. J. Neuropath. exp. Neurol., 15, 119-145.

Van Buren, J. M. (1963). The Retinal Ganglion Cell layer. Charles C. Thomas, Springfield, Illinois.

Vogt, C., and Vogt, O. (1941). Thalamusstudien I-III. J. Psychol. Neurol. (Lpz.), 50, 32-154.

Walker, A. E. (1938). The Primate Thalamus. University of Chicago Press, Chicago.

- (1940). A cytoarchitectural study of the prefrontal area of the macaque monkey. J. comp. Neurol., 73, 59-86.

Walsh, F. B. (1947). Clinical Neuro-ophthalmology. Williams and Wilkins, Baltimore. 